\title{
Are novel objects perceived as stressful? The effect of novelty on heart rate.
}

Clare Parker Fischer ${ }^{1}$, Leor A. Franco ${ }^{1}$, L. Michael Romero ${ }^{1}$

${ }^{1}$ Tufts University, Department of Biology, 163 Packard Ave., Medford, MA, 02155

Total Figures: 6

Abbreviated title: "The effect of novelty on heart rate"

*Correspondence to: Clare Parker Fischer, clare.parker@tufts.edu, Tufts University Department of Biology, 163 Packard Ave., Medford, MA 02155 


\begin{abstract}
Neophobia, or the fear of novel objects, is a behavior that is often found in wild animals. Neophobia appears to be related to the physiological stress response because individuals with higher glucocorticoid responses to stress often are more neophobic. The relationship between the heart rate response and novelty, however, has not been tested in a wild species. We implanted heart rate transmitters in captive European starlings (Sturnus vulgaris) to measure increases in heart rate as an index of the adrenomedullary stress response. Specifically, we measured heart rate in animals encountering novel objects on or near their food dishes using a system to display the novel objects while the experimenters remained outside the room, thereby minimizing the confounding effects of experimenter presence on heart rate. We analyzed three conditions: the period of adjustment to the experimental setup before any exposure to novelty, novel object trials, and no object controls (presented in a random order after 0-5 novel objects). Birds approached their food dishes faster during the adjustment period than during novel object trials. Although they demonstrated a behavioral aversion to novelty, the effect on heart rate was unexpected. Heart rate increased sharply when the food dishes were displayed. The duration of the startle response was longer during no object controls than during novel object exposure, the opposite of the anticipated result. There were no correlations between behavior and metrics of the heart rate response. Novel object exposure does not cause an increase in heart rate.
\end{abstract}

Keywords: neophobia, startle response, heart rate, avian, stress, novelty

\title{
Highlights
}

- We measured the heart rate response to novel objects in European starlings.

- Animals took longer to approach novel objects than their unaltered food dishes.

- Birds approached their dishes more quickly before any exposure to novelty. 
- However, novelty did not cause an increase in the duration of the startle response.

- There were no correlations between behavior and heart rate metrics.

\section{Acknowledgments}

Many thanks to the undergraduates who assisted with data collection: Rohan Rao and Kendra Nowak. Thanks to Devin Merullo and Robert DeBruijn for access to their unpublished data. Thanks to the staff of the Tufts Department of Laboratory Animal Management. This project was supported by NSF grant IOS-1048529 to L.M.R. and NSF REU grant DBI 1263030 to Dr. Philip Starks. Many thanks to Dr. Starks for supporting L.A.F. in her summer research fellowship.. 


\section{Introduction -}

Neophobia, the "fear of the new", is an ecologically relevant behavior characterized by an aversion to novelty, particularly novel objects. To an animal in the wild, a novel object could represent either a threat or a potential untapped resource. A neophobic animal may avoid the risks associated with novelty such as predation (Brown et al., 2013), but may not be able to gain access to new resources (reviewed by Greenberg and Mettke-Hofmann, 2001). Neophobia is often assessed by placing novel objects on or near a food source (Greenberg and Mettke-Hofmann, 2001). The animals must weigh the potential threat of the object against their desire to approach the food. Neophobia has been measured this way in a number of wild bird species in captivity (Apfelbeck and Raess, 2008; Boogert et al., 2006; Greenberg, 1992; Martin and Fitzgerald, 2005) and in the field (Herborn et al., 2010; Schoech et al., 2009). In European starlings (Sturnus vulgaris) (Feenders et al., 2011) and blue tits (Cyanistes caeruleus) (Herborn et al., 2010) neophobic responses are equivalent in the wild and in captivity. Neophobic birds take much longer to approach a novel food dish or novel food type than they do their regular dish or food, but will rapidly habituate and no longer avoid novel objects as they encounter them multiple times (Rankin et al., 2009). Although fear of the new object may drive the behavioral responses, it is unclear whether the birds experience stress as they determine whether to approach an object they have never before encountered. The relationship between fear of novelty and stress is not fully understood.

The physiological stress response is a conserved reaction to a broad range of noxious situations that may be encountered by an animal, such as predator attacks, storms, disease, famine, etc. It consists of three arms. The adrenomedullary response begins in less than a second and consists of the increase in heart rate and mobilization of energy caused by the rapid release of catecholamine hormones (epinephrine and norepinephrine) from the adrenal medulla. The glucocorticoid response begins within minutes and can last for hours. It consists of the release of cortisol (in most mammals) or 
corticosterone (in birds) into the blood. Glucocorticoids have wide-ranging effects on many bodily functions, from energetics to immune function to reproduction to behavior (reviewed in Sapolsky et al., 2000). The third branch of the stress response is the animal's adjustment of its behavior to appropriately confront the stressor. The three branches of the stress response are linked, but still maintain independent regulation from one another. For example, great tits (Parus major) show behavioral aversion to many stimuli, but they only increase glucocorticoid levels in response to some stimuli, i.e. a predator (Cockrem and Silverin, 2002). European starlings independently regulate their behavior, heart rate, and glucocorticoid levels in response to different types of stressful stimuli (de Bruijn and Romero, 2013; Nephew et al., 2003). Neophobia resembles the behavioral arm of the stress response - it is a behavioral response to a stimulus which an animal might perceive as threatening.

The relationship between the glucocorticoid arm of the stress response and neophobia has been assessed in several bird species, with mixed results. The glucocorticoid response to a standardized stressor correlated with the behavioral response to novel objects in free-living Florida scrub jays (Aphelocoma coerulescens) (Schoech et al., 2009), free-living house sparrows (Passer domesticus)(Lendvai et al., 2011), and great tits artificially selected for low neophobia (Baugh et al., 2012). In these studies, birds with strong glucocorticoid responses also had high fear and/or low exploration towards novel objects. However, in free-living collared flycatchers (Ficedula albicollis) (Garamszegi et al., 2012) and zebra finches (Taeniopygia guttata) artificially selected for high and low corticosterone responses (Martins et al., 2007), there was no relationship between glucocorticoids and behavior towards novel objects. Novel objects cause either no or a very small glucocorticoid response in both European starlings (Apfelbeck and Raess, 2008) and Japanese quail (Coturnix japonica) (Richard et al., 2008) even when the birds demonstrate a strong behavioral response. In our previous experiments, an injection of corticosterone had no effect on neophobia in European starlings (unpublished data, D. Merullo and R. DeBruijn). In sum, it appears that there is a connection between 
neophobia and the glucocorticoid response, but it is still unclear whether neophobia activates the glucocorticoid arm of the stress response in birds. Furthermore, the time frame of a neophobic response and glucocorticoid release are not well matched.

In contrast, neophobic behavior and the heart rate response share a similar time frame that is much more rapid than glucocorticoid release, so perhaps neophobia activates the adrenomedullary arm of the stress response. The heart rate response to stress can be measured in European starlings using implantable heart rate transmitters (Cyr et al., 2009; Nephew et al., 2003). Captive starlings show a robust startle response (i.e., a rapid increase in heart rate) within a fraction of a second of being exposed to various types of stressors (Cyr et al., 2009; Nephew et al., 2003). In this study, we tested the hypothesis that novel objects will cause an increased heart rate in starlings.. We measured heart rate in captive wild-caught European starlings during novel object exposure or exposure to the birds' normal food dish. Because the presence of a human causes a strong heart rate response in starlings (Nephew et al., 2003), we created a system to expose hungry animals to either their familiar food dish or a dish modified with a novel object while the experimenter remained outside the room. We hid the food dish in an opaque black box that we lifted by pulling a string that ran outside the room where the birds were housed. When the black box was suddenly lifted, we anticipated that the animals would have a startle response (i.e., a sharp increase in heart rate). We hypothesized that heart rate would take longer to return to baseline when the animals were exposed to a novel object instead of their familiar dish. That is, the combined effect of startle and novelty would be greater than the startle effect alone. We further hypothesized that the animals would approach the food dish at approximately the time that their heart rate returned to baseline.

\section{Materials and Methods}

\subsection{Animals}


Twelve European starlings were caught in March 2014 at a suburban dairy farm in eastern Massachusetts. They were housed in an outdoor aviary until late May 2014. They were then moved to an indoor animal facility and housed in individual cages on a 16L:8D light cycle. They were given at least four weeks to acclimate to the indoor facility. Heart rate could be measured on only four birds at a time. Two groups of four were tested in June-July; one group of four was tested in September. The birds from September had been used as controls in a previous experiment. Feather and blood samples had been taken from these birds one month or more before the beginning of this trial, but otherwise they were only exposed to standard caretaking. Just before experiments began, they were surgically implanted with heart rate transmitters and allowed to recover for several days (following Nephew et al., 2003). Heart rate transmitters were purchased from Data Sciences International (St. Paul, MN). We used two different models, TA 10EA-F20 and TA 11ETA-F10. The transmitters send radio signals to a receiver attached to one side of each bird's cage. The data are then transferred to a computer equipped with Dataquest Advanced Research Technology Gold 4.0 software package, which records continuous ECG signals. The heart rate transmitters have a magnetic switch which could be turned on at the beginning of every experimental day. The birds' weights were monitored 1-2x weekly throughout the experiment. No significant weight changes were observed. We used a mix of males and females. There have been no previous differences found between the stress responses of captive males and females in this species (Nephew and Romero, 2003; Nephew et al., 2003; Nephew et al., 2005). All experiments complied with Association for Assessment of Laboratory Animal Care guidelines and were approved by the Tufts Institutional Animal Care and Use Committee.

\subsection{Remote presentation of objects}

Because we were looking for the heart rate response to novelty, we wanted to reduce the heart rate response to extraneous stimuli as much as possible. Therefore, we constructed a device to allow the animals to be exposed to novel objects without the presence of the researchers (Fig. 1). A 
black box constructed of foam core board was placed over the food dish. It was hinged onto the cage to prevent birds from entering from the top and was weighted with washers to prevent the birds from lifting it up. A string allowed researchers to pull the box up and display the food dish from outside the room. The behaviors of the animals were monitored using video cameras.

\subsection{Neophobia trials}

On the night before each experimental trial, the birds' food dishes were removed overnight to stimulate appetite and ensure the animals would be motivated to approach their food dishes in the morning. Opaque blinders were placed between the cages at the same time to prevent the birds from seeing one another's behavior and the objects the other birds were being exposed to. Starlings show more neophobia in isolation than when they are in contact with other starlings (Apfelbeck and Raess, 2008). However, an individual bird's response to novelty can be affected by the behavior it has observed in others (Fryday and Greig-Smith, 1994). In the morning, birds were caught and their heart rate transmitters were switched on. At the same time, the opaque boxes were placed in the cages over the animals' food dishes (birds were restrained in a cloth bag for $\sim 5$ minutes while boxes were placed). The animals were then given 10 minutes for their heart rate to recover from handling stress. Baseline heart rate was then assessed for 20 minutes (beginning $t=-20$ ). At $t=0$, the box was lifted, exposing birds to their food dish. Behavior and heart rate were monitored for 20 minutes. Heart rate transmitters were then switched off, boxes and novel objects removed from the cage, and the opaque dividers removed from between the cages. The birds were acclimated to this procedure by presenting the normal food dish for at least three days before the start of the experiment. (The first four birds tested in June were given 10 days to adjust; due to time restrictions, the remainder of the birds were given 4-5 days.)

On experiment days 1 through 7, birds were exposed to novel objects in a random order. The objects used were unlikely to resemble anything the birds had previously encountered. Objects used were a food dish painted red, a yellow bottle opener keychain clipped to the cage behind the food 
dish, a colored plastic Easter egg placed in the food dish, a springy red ring wrapped around the food dish, a red ribbon stretched across the top of the food dish, and a white cover placed over the food dish. Many of the objects were red because that has been shown to be a color that more powerfully elicits neophobia (Roper, 1990) and each object had been previously shown to stimulate neophobia in European starlings (unpublished data, D. Merullo and R. DeBruijn). Birds were also shown a no object control (i.e., normal food dish) on one randomly chosen experimental day.

Videos were analyzed for the latency to approach and feed from the dish from the time the box was lifted. Approach was defined as perching on the dish for at least 1 second; feeding was defined by the beak dipping into the dish for at least 2 seconds. Different objects may stimulate neophobia to a greater or lesser degree, but we were not interested in the differences between objects, just in an individual's mean response to novelty. We therefore averaged the approach and feed latency times for each bird across all novel objects.

Heart rate was measured in the animals for 20 minutes before and 20 minutes after object exposure. It was analyzed using the Ponemah Physiology Platform (Data Sciences International, Valley View, $\mathrm{OH}$ ). Heart rate (beats per minute, bpm) was calculated in thirty second intervals using a forward frame (i.e. averaged over the 30 seconds following each time stamp). Some parts of the traces contained breaks in the data or noise which disrupted the signal. The traces were each individually examined, and problematic portions were eliminated manually. Maximum heart rate was determined as the highest bpm after raising the box, and the duration of the startle response was calculated as the time for the heart rate to return to within 1 standard deviation of baseline (Nephew et al., 2003).

\subsection{Analysis}

All statistical analyses were conducted in R (Version 3.1.3; R Core Team). The design for some of our analyses was unbalanced (for example, heart rate traces were sometimes unusable), so linear mixed effects models were constructed using the "lmer" function in the lme4 package of R 
(Bates et al., 2014) with individual bird as a random variable. We then used the "Anova" function in the car package (Fox and Weisberg, 2011) to calculate a Type II Wald F test with Kenward-Roger adjusted degrees of freedom. We followed this with a Tukey's multiple comparison test if warranted. This method was used for all analyses. Approach latency and feed latency were analyzed by averaging the values over all novel object trials and comparing the resultant means to the no object trials, An unanticipated behavioral difference was detected between the adjustment period and the experimental trials. We therefore analyzed behavioral and heart rate metrics (approach latency, feed latency, baseline heart rate, peak heart rate, and duration of startle response) by comparing the adjustment period, the no object controls, and the trials with novel objects. All data are reported as mean \pm standard error.

\section{Results}

\subsection{Behavior}

Of the twelve birds in the study, three birds did not approach the no object control during the experimental period. (See Fig. S1A for individual approach latencies.) These three birds were excluded from behavioral analyses. We compared mean latency to approach the novel dishes with latency to approach the familiar dish and found a significant effect of novelty $(\mathrm{F}=5.48, \mathrm{df}=1, \mathrm{df} . \mathrm{res}=8$, $\mathrm{p}=0.047$, Fig. 2C). Similarly, there was a significant effect of novelty on mean feed latency $(F=6.50$, $\mathrm{df}=1$, df.res $=8$, p=0.03, Fig. 2D). Birds took $362 \pm 123$ seconds (mean \pm se) to approach the familiar dish, and $589 \pm 89$ seconds to approach a novel dish. They fed from the familiar dish after $495 \pm 146$ seconds and from a novel dish after $798 \pm 106$ seconds. Data from all birds (including those that did not approach the no object dish) are presented in Supplemental Fig. S2.

The birds showed a moderate response to novelty. However, they behaved differently during the experimental period compared to when they were adjusting to the experimental setup. Behavior and heart rate was only recorded for the last 1-3 days of the adjustment period (adjustment 
period lasted 4-10 days). Animals took significantly less time to approach during the adjustment period than during the novel object exposure (Overall model: $F=7.07, \mathrm{df}=2$, df.res $=60.03, \mathrm{p}=0.002$; Tukey's: adjust vs. novel object: $\mathrm{z}=3.65$, $\mathrm{p}<0.001$, Fig. $3 \mathrm{~A}$ ). They fed more quickly during the adjustment period than during novel object exposure (Overall model: $\mathrm{F}=6.04$, $\mathrm{df}=2$, df.res=60.07, $\mathrm{p}=0.004$; Tukey's: adjust vs. novel object: $\mathrm{z}=3.24, \mathrm{p}=0.004$, Fig. 3B). The number of times the birds were exposed to the box did not affect the time to approach or feed (Approach: $F=0.72, d f=1$, df.res=27.67, p=0.40, Fig. 3C; Feed: $F=1.53$, df=1, df.res=24.45, p=0.23, Fig. 3D). These patterns held true when the three birds that did not approach during experimental no object trials were included in the analysis (see Supplemental Fig. S3).

\subsection{Heart rate}

Of the twelve birds, only nine had usable heart rate data. There was no difference in baseline heart rate (averaged over the 10 minutes before the box was lifted) throughout the experiment (adjustment period: $338 \pm 13$ bpm; no object control: $343 \pm 18$ bpm; novel dish exposure: $358 \pm 10$ bpm; $\mathrm{F}=1.12$, df=2, df.res=53.68, $\mathrm{p}=0.33$ ). When the box was lifted to expose animals to their food dish, the birds had a strong startle response (Fig. 4). Heart rate sometimes may have increased just before box lift when birds could hear the boxes move in the neighboring cages. There was no significant difference in maximum startle-induced heart rate between the adjustment period, the no object controls, and the novel object exposures $(\mathrm{F}=1.74, \mathrm{df}=2$, df.res=53.9, $\mathrm{p}=0.19$, Fig. 5A $)$. There was a significant difference in the duration of the startle response between adjustment, no object, and novel object $(\mathrm{F}=3.65, \mathrm{df}=2$, df.res $=55.41, \mathrm{p}=0.03$, Fig. $5 \mathrm{~B})$. During the no object trials, duration was marginally longer than during the adjustment period $(\mathrm{z}=2.21, \mathrm{p}=0.07)$ and significantly longer than during the novel object exposure $(\mathrm{z}=-2.66, \mathrm{p}=0.02)$. There was no difference between the adjustment period and novel object exposure ( $\mathrm{z}=-0.095, \mathrm{p}=1)$. (See Fig. S1B for individual startle durations.)

Approach time and feed time were unrelated to the duration of the startle response 
(Approach: $F=1.37, \mathrm{df}=1$, df.res=57.59, p=0.25, Fig. 6A; Feed: $F=1.06$, df=1, df.res=58.60, $p=0.31$, Fig. 6B). Approach and feed times were also unrelated to maximum heart rate (Approach: $F=0.35$, $d f=1$, df.res=61.82, p=0.56, Fig. 6C; Feed: $F=0.02$, df=1, df.res=62, p=0.88, Fig. 6D). On average, the startle response was completed more than a minute before the birds approached the food dish (Fig. 4).

\section{Discussion}

Neophobia is an ecologically relevant behavior in wild birds. Different ecological and behavioral contexts require a different balance of caution and boldness. Individuals within a species or across species use different strategies and therefore have different levels of neophobia. Less fear may be more beneficial for species which employ a generalist strategy (i.e., those which eat a broad variety of foods) or are currently invading new territory (which contains unknown food sources), as they will come into contact with novelty more frequently and have more to gain by seeking out new resources (Echeverria et al., 2006; Greenberg and Mettke-Hofmann, 2001). For example, house sparrows that are currently invading new territory demonstrate less neophobia than house sparrows which have occupied the same region for several generations (Martin and Fitzgerald, 2005). Similarly, the habitat-generalist song sparrows are less neophobic than their specialist relatives, the swamp sparrow (Greenberg, 1990; Greenberg, 1992). Individuals tend to adapt a stable behavioral strategy for coping with the environment. Neophobia is a key part of these strategies. Individual birds tend to show repeatable levels of neophobia when shown different novel objects (Greenberg, 1990; Schoech et al., 2009). Some measure of neophobia is commonly used when assessing different personalities which are stable in different contexts (Boogert et al., 2006; Drent et al., 2003). Nevertheless, the physiology behind the behavior is not fully understood. Does avoidance of novel objects indicate that an individual finds their presence stressful?

A few previous experiments have examined the relationship between the heart rate 
response and neophobia. Horses exposed to novel audiovisual stimuli (a film being played while the animals exercised on a familiar treadmill) showed an increase in heart rate as well as increased ACTH (a hormone involved in the glucocorticoid pathway) (Hada et al., 2003). Rats showed an increase in heart rate when exposed to the novel environment of an open field (van den Buuse et al., 2001), although much of the difference in heart rate can be explained by a change in wakefulness - the nocturnal animals were exploring the field in the middle of the day during a time in which they are normally asleep (Beerling et al., 2011). Two lines of great tits (Parus major) have been artificially selected for high or low levels of neophobia (Drent et al., 2003). The high neophobia line had a higher breath rate in the hand than the low neophobia line, which provides a rough estimation of heart rate during a stress response (Carere et al., 2001). Our current study is the first test of object neophobia in a wild bird species that measured heart rate changes.

Measuring heart rate responses to new objects in wild birds presented some unique challenges. In previous experiments, novel objects were presented directly to the animal by the experimenter (Boogert et al., 2006; Greenberg, 1992; Herborn et al., 2010). However, that was unfeasible in our study - any subtle effect the novel object had on heart rate could be masked by the strong effect of experimenter presence (Cyr et al., 2009). We therefore used a black opaque box to hide the food dish and novel objects from the birds while we recorded baseline heart rate. The box was lifted up with a string from outside the room at $\mathrm{t}=0$, leaving the box dangling over the food dish. The movement of the box itself caused a heart rate response. However, its use eliminated some sources of variability that would be caused by the experimenter directly presenting the food dishes (eg. identity of the experimenter, length of time in the room, amount of time to position the objects.) During the adjustment period, every bird was observed to approach and feed at least once.

The animals in our study showed only moderate neophobia. Three birds never approached their food dish in the twenty minute observation period during experimental trials. These individuals 
were likely temperamentally different from the other nine birds. Individuals can vary in the intensity of their neophobic responses, at least in house sparrows (Ensminger and Westneat, 2012). When these three birds were removed from behavioral analyses, we could detect a small behavioral difference between the no object controls and the experimental controls. However, a much more robust behavioral difference was found between the initial period of adjustment to the box and the experimental trials. The birds approached and fed with the box much more readily during the last few days of the adjustment period than they did during novel object exposure, a result that was robust whether or not the three temperamentally different birds were included (Figs. 3 and S3). During the adjustment period, many animals appeared unafraid of the box and tried to get inside (personal observation). Because of their persistent efforts, the box had to be weighted heavily with metal washers to prevent them from moving it. However, once they had been exposed to novelty, they perhaps became more wary of the setup as a whole. Some birds discriminated between no object and novel object even after being exposed to novelty. Other birds took longer to approach even the no object controls once they had been exposed to a novel dish - blunting the effect of novelty on behavior. The birds may have learned to be cautious of the box once a novel dish had been revealed - it was no longer certain what would be revealed when it was lifted up. The number of times the birds were exposed to the box did not affect their behavior, either during the adjustment period or during experimental trials. However, the first exposure to the box was never recorded.

In addition to the box in their cages, the birds were also exposed to the stress of handling before the experiments. Although neophobia does not appear to be stressful in itself, the stress response can affect neophobia. In previous trials using European starlings, exposure to restraint stress before novel object exposure increased the latency to approach the object (unpublished data, D. Merullo and R. DeBruijn). Because of the procedure for measuring heart rate, the animals in this study necessarily experienced stress before novel object exposure, which may have resulted in more intense neophobia. 
The stress of handling may have increased the learned fear of the box once novelty had been experienced. Elevated corticosterone has been implicated in learning and memory. For example, mountain chickadees (Poecile gambeli) with chronic but moderate elevation of corticosterone had improved spatial learning (Pravosudov, 2003). Handling may therefore have been partly responsible for the increase in latency to approach the no object controls during the experimental period. Increased corticosterone levels resulting from handling may have helped the animals learn to fear the box once it was shown to be unpredictable by the introduction of novelty.

Of the twelve birds, we were able to get usable heart rate traces from nine. We included all birds in the heart rate analysis whether they approached the no object controls or not because the more robust behavioral difference was between the adjustment period and novel object exposure, a difference that was observed in all birds. The birds all had a startle response when the box was lifted. We measured the combined effect of startle and novelty - that is, how novel objects affected the duration and magnitude of the startle response. Despite the behavioral differences between the trials, the strength of the heart rate response was not different between the adjustment period, the novel dish exposure, and the experimental no object exposure. The startle response lasted an average of 2 minutes during the adjustment period or novel object exposure. However, during the no object control trials, heart rate took longer to return to baseline, about 3.3 minutes. The birds that did not approach the dish during the no object trials, or that had a longer latency to approach than during novel object trials, tended to have particularly high heart rates during no object trials (see birds 446.M, 481.M and 493.M in Supplemental Fig. S1). For some individuals, encountering a normal dish when they expected to see a novel object may have led to a stronger fear response. We did not have a large enough sample size to address the question of why some animals were more fearful of the no object dish than others. On average, by the time the animals approached the food dish, their startle response had been completed for several minutes. There was no correlation between the duration of the startle response and the time 
to approach or feed from the food dish. There was also no correlation between the maximum heart rate and either metric of behavior.

In conclusion, we did not find a strong neophobic response in these birds. However, exposure to novelty affected the birds' behavior - once exposed to a novel object, they approached their dishes more slowly. We anticipated that the birds would show a startle response (increased heart rate) when they were exposed to their food dishes, but hypothesized that the duration of the startle response would be longer with a novel object then without one, corresponding to a longer latency to approach novel food dishes. If the heart rate response is related to the behavior of neophobia, we would expect the startle response and the fear of the food dish to resolve at approximately the same time. We rejected this hypothesis. The heart rate response to startle was completed well before the animals approached and fed from their dishes and there were no correlations between startle response metrics and behavior. It thus appears that captive European starlings do not have a robust adrenomedullary stress response when exhibiting neophobic behavior. 


\section{References}

Apfelbeck, B., Raess, M., 2008. Behavioural and hormonal effects of social isolation and neophobia in a gregarious bird species, the European starling (Sturnus vulgaris). Horm Behav. 54(3), 435-441. DOI: 10.1016/j.yhbeh.2008.04.003

Bates, D., Maechler, M., Bolker, B., Walker, S., 2014. lme4: Linear mixed-effects models using Eigen and S4. R package version 1. 1-7, <URL: http://CRAN.R-project.org/package=lme4>.

Baugh, A.T., Schaper, S.V., Hau, M., Cockrem, J.F., de Goede, P., van Oers, K., 2012. Corticosterone responses differ between lines of great tits (Parus major) selected for divergent personalities. Gen Comp Endocrinol 175(3), 488-494. DOI: 10.1016/j.ygcen.2011.12.012

Beerling, W., Koolhaas, J.M., Ahnaou, A., Bouwknecht, J.A., de Boer, S.F., Meerlo, P., Drinkenburg, W.H.I.M., 2011. Physiological and hormonal responses to novelty exposure in rats are mainly related to ongoing behavioral activity. Physiol Behav 103 (3-4), 412-420. DOI:

10.1016/j.physbeh.2011.03.014

Boogert, N.J., Reader, S.M., Laland, K.N., 2006. The relation between social rank, neophobia and individual learning in starlings. Anim Behav 72(6), 1229-1239. DOI:

10.1016/j.anbehav.2006.02.021

Brown, G.E., Ferrari, M.C., Elvidge, C.K., Ramnarine, I., Chivers, D.P., 2013. Phenotypically plastic neophobia: a response to variable predation risk. Proc R Soc B 280(1756), 20122712. DOI: 10.1098/rspb.2012.2712

Carere, C., Welink, D., Drent, P., Koolhaas, J., Groothuis, T., 2001. Effect of social defeat in a territorial bird (Parus major) selected for different coping styles. Physiol Behav 73(3), 427-433. DOI: 10.1016/S0031-9384(01)00492-9

Cockrem, J.F., Silverin, B., 2002. Sight of a predator can stimulate a corticosterone response in the great tit (Parus major). Gen Comp Endocrinol 125(2), 248-255. DOI: 10.1006/gcen.2001.7749

Cyr, N.E., Dickens, M.J., Romero, L.M., 2009. Heart rate and heart-rate variability responses to acute and chronic stress in a wild-caught passerine bird. Physiol Biochem Zool 82(4), 332-344. DOI: $10.1086 / 589839$

de Bruijn, R., Romero, L.M., 2013. Artificial rain and cold wind act as stressors to captive molting and non-molting European starlings (Sturnus vulgaris). Comp. Biochem. Physiol. A-Mol. Integr. Physiol. 164(3), 512-519. DOI: 10.1016/j.cbpa.2012.12.017

Drent, P., van Oers, K., van Noordwijk, A., 2003. Realized heritability of personalities in the great tit (Parus major). Proc R Soc B 270(1510), 45-51. DOI: 10.1098/rspb.2002.2168

Echeverria, A.I., Vassalo, A.I., Isacch, J.P., 2006. Experimental analysis of novelty responses in a bird assemblage inhabiting a suburban marsh. Can J Zool 84(7), 974-980. DOI: 10.1139/z06-089

Ensminger, A.L., Westneat, D.F., 2012. Individual and sex differences in habituation and neophobia in 
house sparrows (Passer domesticus). Ethology 118(11), 1085-1095. DOI: 10.1111/eth.12009

Feenders, G., Klaus, K., Bateson, M., 2011. Fear and exploration in European starlings (Sturnus vulgaris): A comparison of hand-reared and wild-caught birds. PLoS One 6(4), e19074. $\quad$ DOI: 10.1371/journal.pone.0019074

Fox, J., Weisberg, S., 2011. An \{R\} Companion to Applied Regression. Sage, Thousand Oaks, CA.

Fryday, S.L., Greig-Smith, W., 1994. The effects of social learning on the food choice of the house sparrow (Passer domesticus). Behaviour 128(3), 281-300. DOI: 10.1163/156853994X00299

Garamszegi, L.Z., Rosivall, B., Rettenbacher, S., Markó, G., Zsebők, S., Szöllősi, E., Eens, M., Potti, J., and Török, J. 2012. Corticosterone, avoidance of novelty, risk-taking and aggression in a wild bird: No evidence for pleiotropic effects. Ethology 118 (7), 621-635. DOI: 10.1111/j.14390310.2012.02049.x

Greenberg, R., Mettke-Hofmann, C., 2001. Ecological aspects of neophobia and neophilia in birds. In Current Ornithology, Volume 16, 119-178.

Greenberg, R., 1990. Feeding neophobia and ecological plasticity: a test of the hypothesis with captive sparrows. Anim Behav 39(2), 375-379. DOI:10.1016/S0003-3472(05)80884-X

Greenberg, R., 1992. Differences in neophobia between naive song and swamp sparrows. Ethology 91(1), 17-24. DOI: 10.1111/j.1439-0310.1992.tb00847.x

Hada, T., Onaka, T., Takahashi, T., Hiraga, A., Yagi, K., 2003. Effects of novelty stress on neuroendocrine activities and running performance in thoroughbred horses. J Neuroendocrinol 15(7), 638-648. DOI: 10.1046/j.1365-2826.2003.01042.x

Herborn, K.A., Macleod, R., Miles, W.T.S., Schofield, A.N.B., Alexander, L., Arnold, K.E., 2010. Personality in captivity reflects personality in the wild. Anim Behav 79(4), 835-843. DOI: 10.1016/j.anbehav.2009.12.026

Lendvai, A.Z., Bokony, V., Chastel, O., 2011. Coping with novelty and stress in free-living house sparrows. J Exp Biol 214(5), 821-828. DOI: 10.1242/jeb.047712

Martin, L.B., Fitzgerald, L., 2005. A taste for novelty in invading house sparrows, Passer domesticus. Behav Ecol 16(4), 702-707. DOI: 10.1093/beheco/ari044

Martins, T.L.F., Roberts, M.L., Giblin, I., Huxham, R., Evans, M.R., 2007. Speed of exploration and risk-taking behavior are linked to corticosterone titres in zebra finches. Horm Behav 52(4), 445453. DOI: 10.1016/j.yhbeh.2007.06.007

Nephew, B., Romero, L., 2003. Behavioral, physiological, and endocrine responses of starlings to acute increases in density. Horm Behav 44(3), 222-232. DOI: 10.1016/j.yhbeh.2003.06.002

Nephew, B.C., Aaron, R.S., Romero, L.M., 2005. Effects of arginine vasotocin (AVT) on the behavioral, cardiovascular, and corticosterone responses of starlings (Sturnus vulgaris) to crowding. Horm Behav 47(3), 280-289. DOI: 10.1016/j.yhbeh.2004.11.007 
Nephew, B.C., Kahn, S.A., Romero, L.M., 2003. Heart rate and behavior are regulated independently of corticosterone following diverse acute stressors. Gen Comp Endocrinol 133(2), 173-180. DOI: 10.1016/S0016-6480(03)00165-5

Pravosudov, V., 2003. Long-term moderate elevation of corticosterone facilitates avian food-caching behaviour and enhances spatial memory. Proc R Soc B-Biol Sci 270, 2599-2604.

R Core Team. 2015. R: A language and environment for statistical computing. R Foundation for Statistical Computing, Vienna, Austria. <URL: http://www.R-project.org/>.

Rankin, C.H., Abrams, T., Barry, R.J., Bhatnagar, S., Clayton, D.F., Colombo, J., Coppola, G., Geyer, M.A., Glanzman, D.L., Marsland, S., McSweeney, F.K., Wilson, D.A., Wu, C., Thompson, R.F., 2009. Habituation revisited: An updated and revised description of the behavioral characteristics of habituation. Neurobiol Learn Mem 92(2), 135-138. DOI: 10.1016/j.nlm.2008.09.012

Richard, S., Wacrenier-Cere, N., Hazard, D., Saint-Dizier, H., Arnould, C., Faure, J.M., 2008. Behavioural and endocrine fear responses in Japanese quail upon presentation of a novel object in the home cage. Behav Processes 77(3), 313-319. DOI: 10.1016/j.beproc.2007.07.005

Roper, T.J., 1990. Responses of domestic chicks to artificially coloured insect prey: effects of previous experience and background colour. Anim Behav 39(3), 466-473. DOI: 10.1016/S00033472(05)80410-5

Sapolsky, R.M., Romero, L.M., Munck, A.U., 2000. How do glucocorticoids influence stress responses? Integrating permissive, suppressive, stimulatory, and preparative actions. Endocr Rev 21(1), 55-89. DOI: 10.1210/edrv.21.1.0389

Schoech, S.J., Rensel, M.A., Bridge, E.S., Boughton, R.K., Wilcoxen, T.E., 2009. Environment, glucocorticoids, and the timing of reproduction. Gen Comp Endocrinol 163(1-2), 201-207. DOI: 10.1016/j.ygcen.2008.09.009

van den Buuse, M., van Acker, S.A.B.E., Fluttert, M., de Kloet, E.R., 2001. Blood pressure, heart rate, and behavioral responses to psychological "novelty" stress in freely moving rats.

Psychophysiology 38(3), 490-499. DOI: 10.1111/1469-8986.3830490 
Figure 1. Device to remotely show novel objects. Researchers can lift up the opaque black box by pulling a string from outside the room.

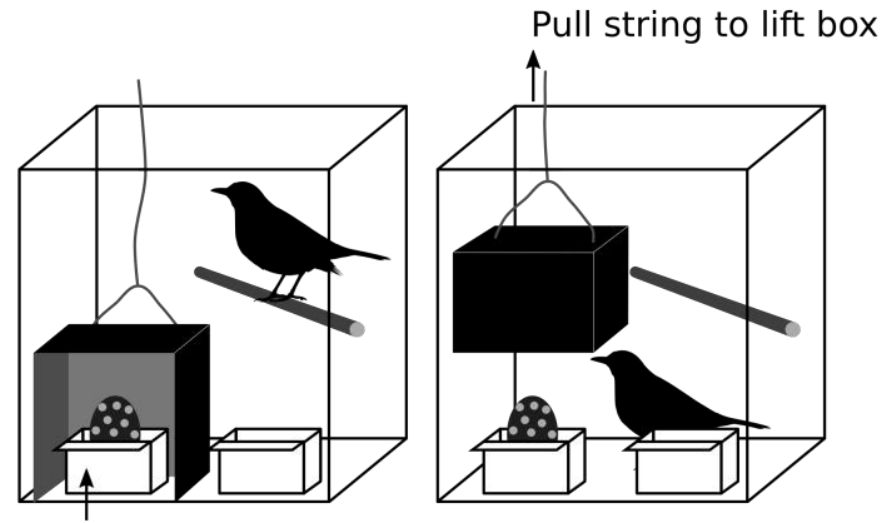

Food dish containing novel object.
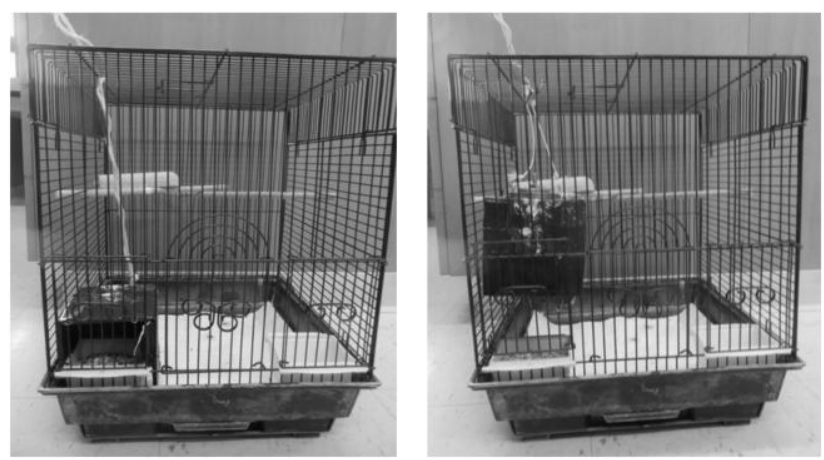
Figure 2. A) Time to approach the feed dish for the no object control vs the mean time to approach novel object exposure for each bird. B) Time to feed from the dish for the no object control vs. the mean time during novel object exposure for each bird. Error bars indicate mean+SE. Data are from the birds that approached the unaltered dish. Data for all birds is shown in supplemental Fig. S2.
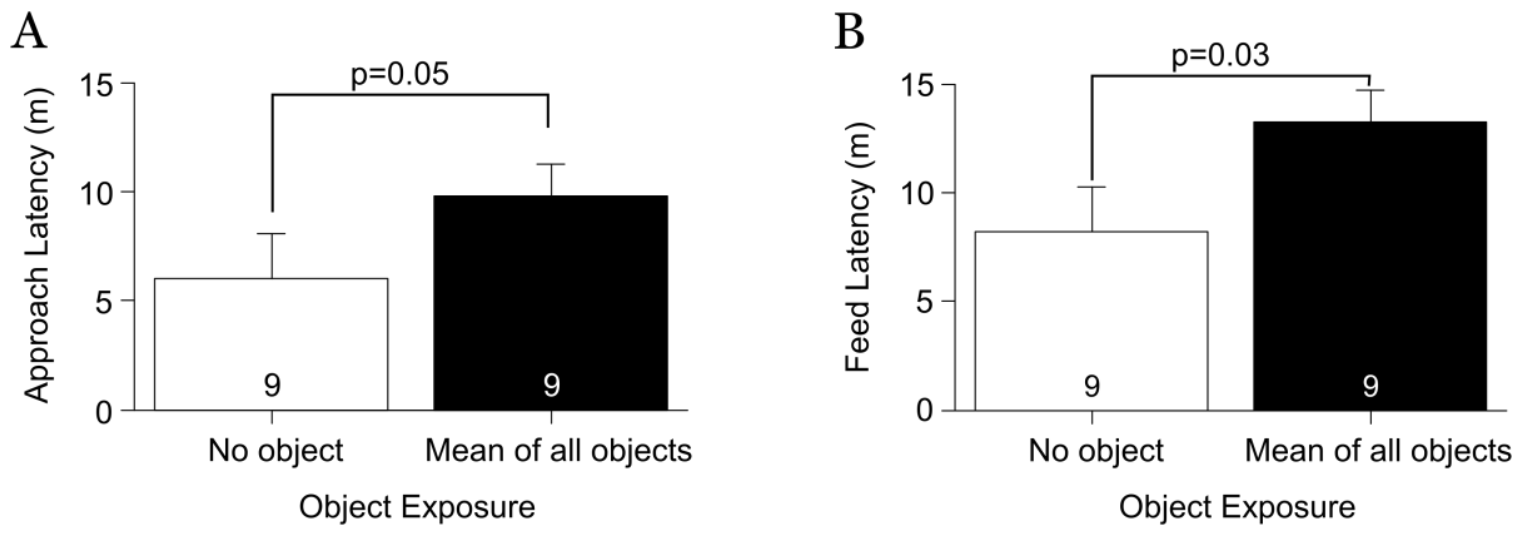
Figure 3. (A) Birds took longer to approach the feed dish during novel object exposure than during the last three days of the adjustment period. (B) Birds took longer to feed from the dish during novel object exposure than during the adjustment period. Error bars indicate mean + SE. Different letters indicate significant differences. There is no relationship between approach time (C) or feed time (D) and the number of times exposed during the adjustment period or the experimental period. The first time the birds were exposed to the box was never recorded. Data are from the birds that approached the unaltered dish. Data for all birds is shown in supplemental Fig. S3.
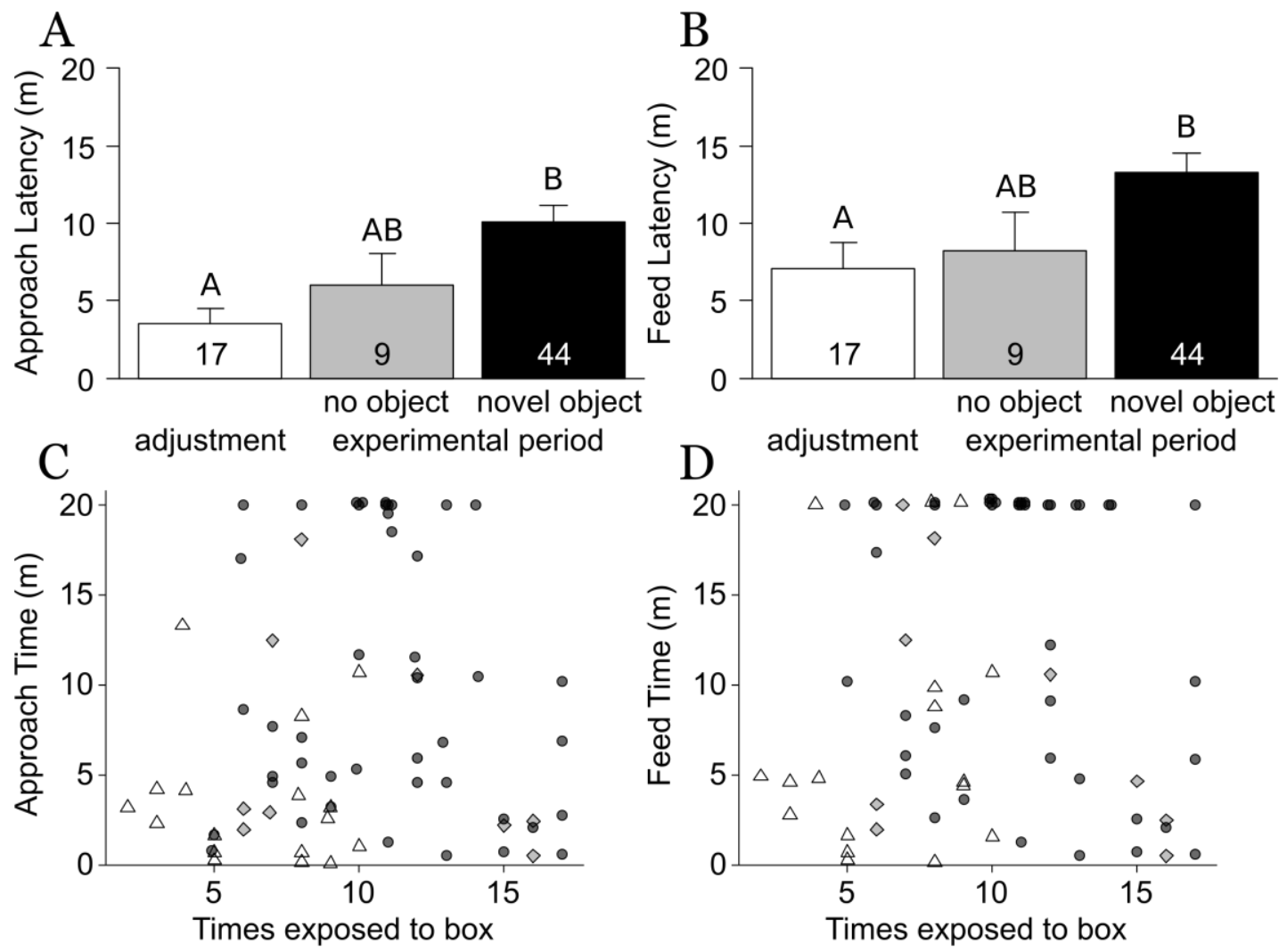

$\Delta$ adjustment period $\diamond$ no object $\bullet$ novel object 
Figure 4. Heart rate during the adjustment period, the no object controls, or exposure to novel objects. Birds were exposed to the food dish at $\mathrm{t}=0$. Arrows indicate the mean time for heart rate to return to baseline (startle duration) and mean time to approach the food dish. Startle duration during the adjustment period and novel object exposure were equivalent. Birds approached the novel object well after the end of the startle response. Heart rate was averaged using a "forward" frame - i.e. average of 30 seconds following each time point.

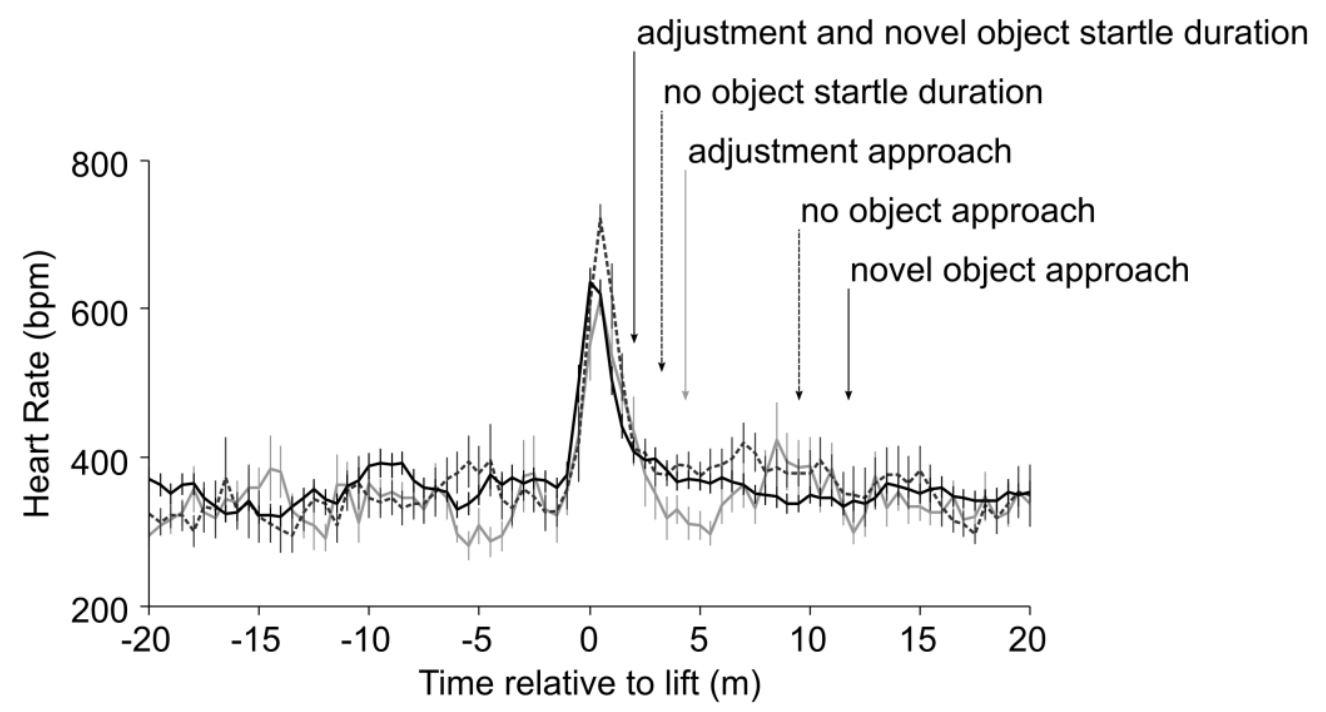

— adjustment period -.--no object $\quad$ novel object 
Figure 5. Startle response during the adjustment period, exposure to the normal food dish, or food dish plus novel object. A) Highest heart rate after dish exposure. B) Time for heart rate to return to within 1 $\mathrm{SD}$ of baseline. Different letters indicate significant differences. Error bars indicate mean $\pm \mathrm{SE}$.
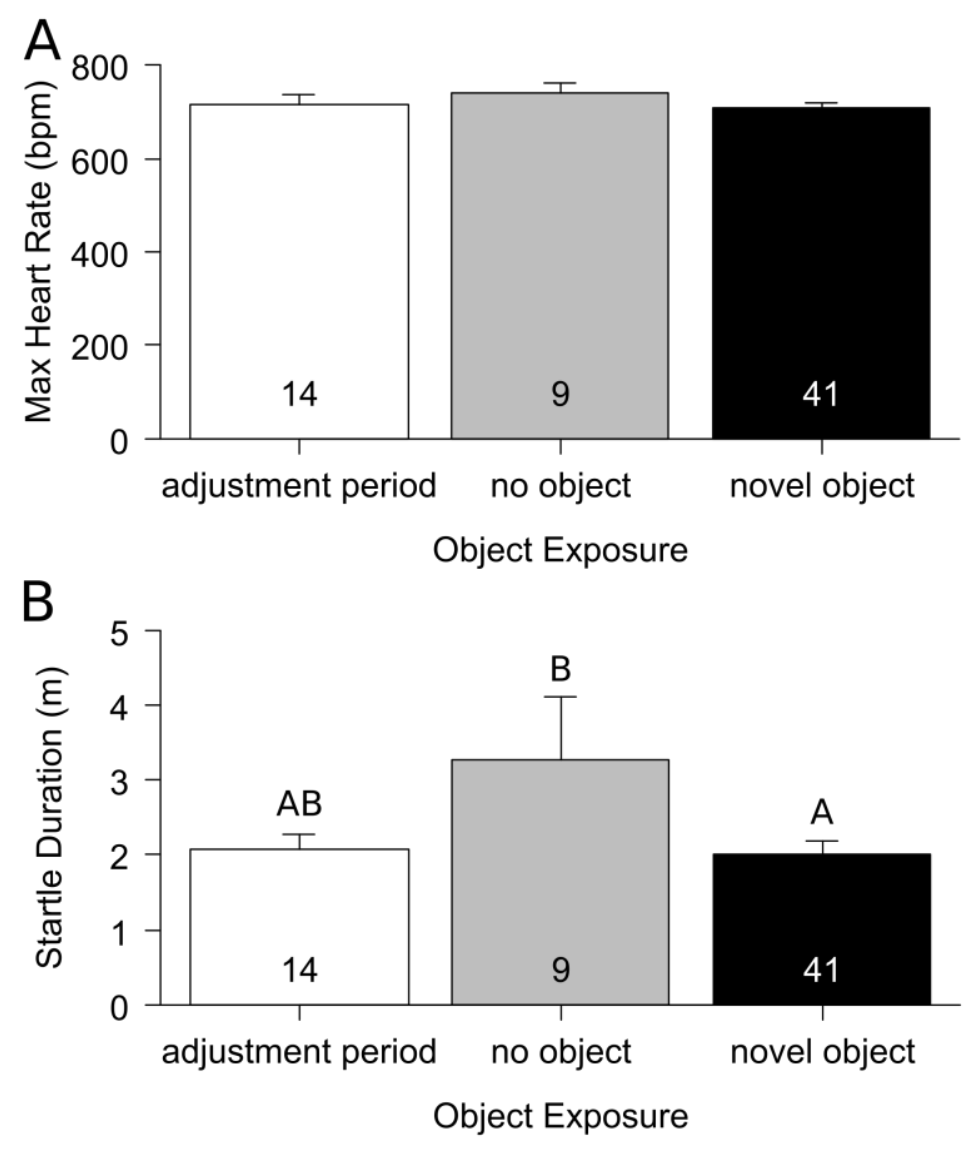
Figure 6. There is no relationship between heart rate response and behavior. Heart rate response duration (time to return to baseline after startle) vs. approach time (A) and feed time (B). Maximum heart rate after startle vs. approach time (C) and feed time (D).

A
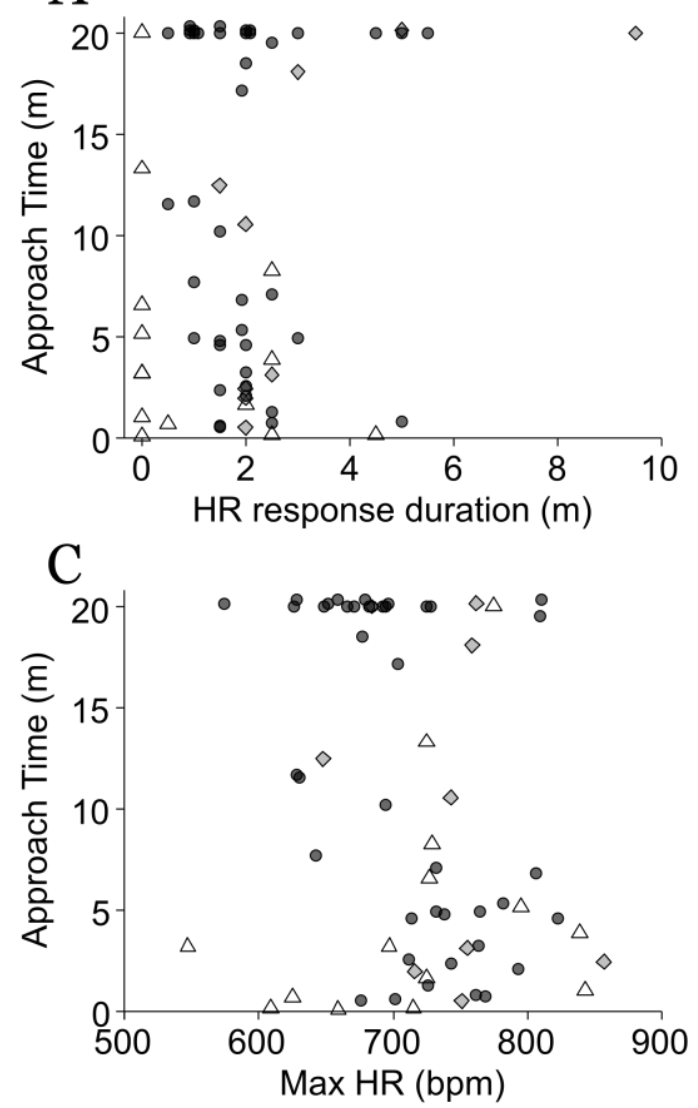
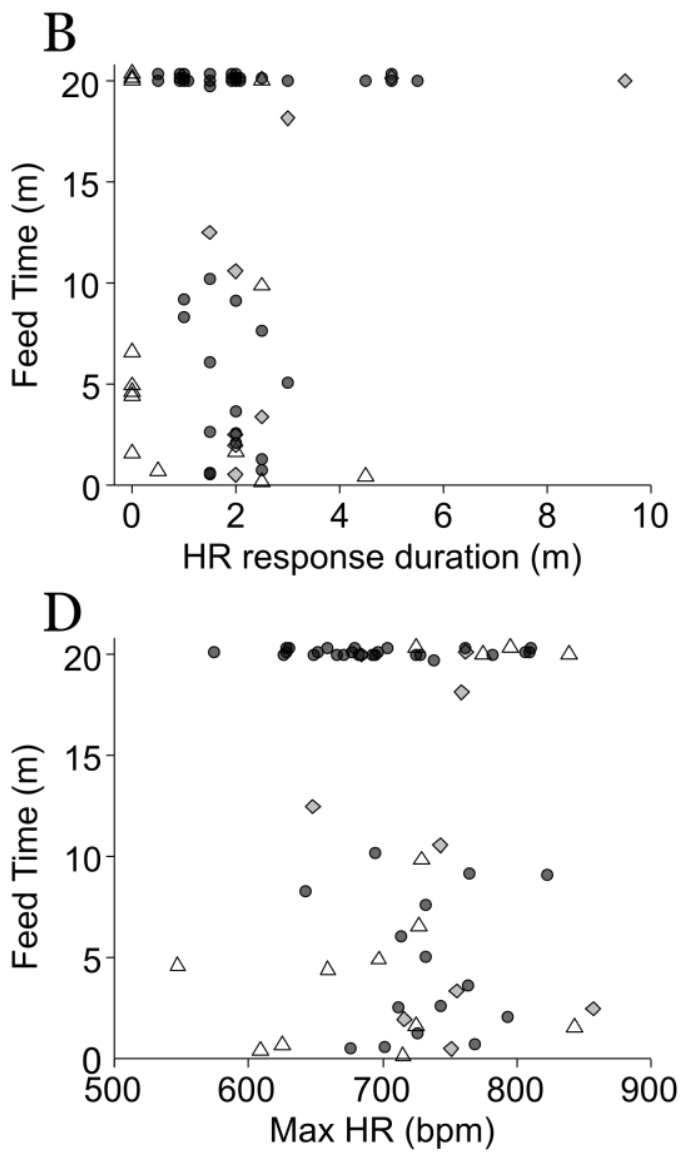

$\Delta$ adjustment period $\diamond$ no object $\bullet$ novel object 